\title{
Reinforcing Effects of Binary Mixtures of Common Bath Salt Constituents: Studies with 3,4-Methylenedioxypyrovalerone (MDPV), 3,4-Methylenedioxymethcathinone (Methylone), and Caffeine in Rats
}

\author{
Brenda M Gannon', Kayla I Galindo', Melson P Mesmin', Kenner C Rice ${ }^{2}$ and Gregory T Collins*,1,3 \\ 'Department of Pharmacology, University of Texas Health Science Center at San Antonio, San Antonio, TX, USA; ${ }^{2}$ Molecular Targets and \\ Medications Discovery Branch, NIDA and NIAAA, Bethesda, MD, USA; ${ }^{3}$ South Texas Veterans Health Care System, San Antonio, TX, USA
}

\begin{abstract}
'Bath salts' use is associated with high rates of abuse, toxicity, and death. bath salt preparations often contain mixtures of drugs including multiple synthetic cathinones (eg, 3,4-methylenedioxypyrovalerone (MDPV) or 3,4-methylenedioxymethcathinone (methylone)) or synthetic cathinones and caffeine; however, little is known about whether interactions among bath salt constituents contribute to the abuserelated effects of bath salts preparations. This study used male Sprague-Dawley rats responding under a progressive ratio schedule to quantify the reinforcing effectiveness of MDPV, methylone, and caffeine, administered alone and as binary mixtures $(n=12$ per mixture). Each mixture was evaluated at four ratios $\left(10: 1,3: 1,1: 1\right.$, and $1: 3$ ) relative to the mean $\mathrm{ED}_{50}$ for each drug alone. Dose-addition analyses were used to determine the predicted, additive effect for each dose pair within each drug mixture. MDPV, methylone, and caffeine maintained responding in a dose-dependent manner, with MDPV being the most potent and effective, and caffeine being the least potent and effective of the three bath salts constituents. High levels of responding were also maintained by each of the bath salts mixtures. Although the nature of the interactions tended toward additivity for most bath salts mixtures, supra-additive (3:1 MDPV:caffeine, and $3: 1$ and $1: 1$ methylone: caffeine) and sub-additive ( $3: 1,1: I$, and I:3 MDPV:methylone) interactions were also observed. Together, these findings demonstrate that the composition of bath salts preparations can have an impact on both their reinforcing potency and effectiveness, and suggest that such interactions among constituent drugs could contribute to the patterns of use and effects reported by human bath salts users. Neuropsychopharmacology (2018) 43, 76I-769; doi:I0.1038/npp.2017.141; published online 2 August 2017
\end{abstract}

\section{INTRODUCTION}

Abuse of 'designer drugs' such as synthetic derivatives of cathinone has become a serious public health problem worldwide. These synthetic cathinones were marketed as safe and legal alternatives to illicit stimulants and are often sold as 'bath salts' preparations; however, increases in poison control center calls and emergency room visits (Substance Abuse and Mental Health Services Administration, 2013) has led to the Drug Enforcement Administration placing 13 of the most commonly detected synthetic cathinones under Schedule I regulations. Frequently sold as powders or capsules, bath salts are often administered via intravenous (IV), oral, or nasal routes with users often administering multiple doses within a session (Forrester, 2012; Baumann et al, 2013; Johnson and Johnson, 2014). Analysis of bath salts preparations obtained in the US suggests that 3,4-methylenedioxypyrovalerone

\footnotetext{
*Correspondence: Dr GT Collins, Department of Pharmacology, University of Texas Health Science Center at San Antonio, 7703 Floyd Curl Drive, MC\# 7764, San Antonio, TX 78229, USA, Tel: + I 210567 4199, Fax: + | 210567 |581, E-mail: CollinsG@uthscsa.edu

Received I3 March 20 17; revised 26 June 20 17; accepted 29 June 20 I7; accepted article preview online 5 July 2017
}

(MDPV) and 3,4-methylenedioxymethcathinone (methylone) were two of the most widely available and abused synthetic cathinones when these drugs first emerged (Spiller et al, 2011; Shanks et al, 2012; Seely et al, 2013). Analogous to other abused stimulants, MDPV and methylone interact with dopamine, norepinephrine, and serotonin transporters (DAT, NET, and SERT, respectively) where MDPV functions as a DAT-selective cocaine-like transporter inhibitor and methylone functions as a non-selective amphetamine-like transporter substrate (Baumann et al, 2013; Eshleman et al, 2013; Simmler et al, 2013).

Bath salts preparations frequently contain multiple psychoactive compounds, including mixtures of multiple synthetic cathinones or a synthetic cathinone and caffeine, and the composition of these preparations varies not only with regard to the identity and purity of the psychoactive ingredient(s), but also varies across time within a single 'brand' (Brandt et al, 2010; Davies et al, 2010; Spiller et al, 2011; Shanks et al, 2012; Caudevilla-Gálligo et al, 2013; Seely et al, 2013; Zuba and Byrska, 2013). Caffeine is also commonly identified in combination with other stimulant drugs (eg, cocaine, 3,4-methylenedioxymethamphetamine (MDMA) and methamphetamine; Goh et al, 2008; 
Lapachinske et al, 2015; Vidal Giné et al, 2016). One study that analyzed the contents of seized drug preparations found that MDPV, methylone, and caffeine were three of the most frequently detected constituents, regardless of formulation (eg, pills, powders, and so on), and that $65-80 \%$ of pills contained both MDPV and caffeine (Seely et al, 2013). Perhaps relatedly, human bath salts users report effects that range from highly pleasurable (eg, euphoria and alertness) to aversive (eg, agitation, paranoia, tachycardia, and death) (Ross et al, 2011; Spiller et al, 2011; Forrester, 2012; Johnson and Johnson, 2014). Although bath salts preparations typically contain more than one psychoactive ingredient, most preclinical research has focused on characterizing the effects of individual cathinones, rather than modeling bath salts as drug mixtures.

Thus, the primary goal of the current study was to determine whether the reinforcing effects of MDPV and methylone are altered when they are administered as binary mixtures with other common bath salts constituents (ie, MDPV+caffeine, methylone+caffeine, and MDPV+ methylone). Although the mechanism of action of caffeine (adenosine $\mathrm{A} 1$ and $\mathrm{A} 2 \mathrm{~A}$ receptor antagonist) differs from stimulants like MDPV and methylone, caffeine has cocainelike and methamphetamine-like discriminative stimulus effects (Garrett and Griffiths, 2001; Justinova et al, 2003; 2009; Collins et al, 2016). In addition, pretreatment with caffeine has been shown to increase/enhance cocaine selfadministration in both rats and monkeys (Horger et al, 1991; Schenk et al, 1994; Comer and Carroll, 1996). Although these findings suggest that caffeine might do more than mimic the effects of drugs such as cocaine or methamphetamine, relatively little is known about the reinforcing effects of drug mixtures containing cocaine and caffeine. Similarly, despite the fact that bath salts preparations often contain caffeine, it is currently unknown whether caffeine alters the reinforcing effects of synthetic cathinones, such as MDPV and methylone.

Dose-addition analyses are a powerful approach to determine the nature of drug-drug interactions (eg, additive, supra-additive, or sub-additive) when both drugs produce the same pharmacologic effect. Because the composition of bath salts preparations varies greatly and because the nature of drug interactions is known to vary depending upon the proportion at which the constituents are mixed, each binary bath salts mixture was evaluated at four fixed-dose ratios $(10: 1,3: 1,1: 1$, and $1: 3)$ relative to the $\mathrm{ED}_{50}$ of each drug. Dose-addition analyses were used to test the general hypothesis that the reinforcing effects (potency and effectiveness) of bath salts mixtures containing multiple synthetic cathinones or a synthetic cathinone and caffeine are greater than would be predicted for an additive interaction.

\section{MATERIALS AND METHODS}

\section{Subjects}

Male Sprague-Dawley rats (275-300 g) were obtained from Harlan (Indianapolis, IN) and maintained in a temperatureand humidity-controlled environment, on a 10/14-h dark/ light cycle. All rats were singly housed and had free access to tap water and Purina rat chow throughout the study. All experimental procedures were conducted in accordance with the Institutional Animal Care and Use Committee of the University of Texas Health Science Center at San Antonio, and the Eighth Edition of the Guide for Care and Use of Laboratory Animals (National Research Council, 2011).

\section{Surgery}

Rats were prepared with chronic indwelling catheters in the left femoral vein under $2 \%$ isoflurane anesthesia as previously described (Gannon et al, 2017a). Penicillin G (60 $000 \mathrm{U} /$ rat) was administered subcutaneously immediately following surgery to prevent infection, and all rats were allowed 5-7 days to recover before commencement of experiments. Catheters were flushed daily with $0.2 \mathrm{ml}$ saline (before operant sessions) and $0.5 \mathrm{ml}$ heparinized saline (100 U/ml, after operant sessions).

\section{Apparatus}

All operant sessions were conducted in standard operant conditioning chambers (Med Associates, St Albans, VT) located inside sound-attenuating cubicles. A set of green, yellow, and red LED lights was located above each of two levers, and a white house light was located at the top center of the opposite wall. A variable speed syringe driver delivered drug solutions through Tygon tubing connected to a stainless steel fluid swivel and spring tether held in place by a counterbalanced arm.

\section{Self-Administration}

Training. Rats were trained to respond under a fixed ratio (FR) 1 schedule of reinforcement for MDPV $(0.032 \mathrm{mg} / \mathrm{kg} / \mathrm{inf})$ or methylone $(0.32 \mathrm{mg} / \mathrm{kg} / \mathrm{inf})$ during daily 90 -min sessions. A yellow LED above the active lever (left or right; counterbalanced) signaled drug availability, and completion of the response requirement resulted in a drug infusion $(0.1 \mathrm{ml} / \mathrm{kg}$ over $\sim 1 \mathrm{~s}$ ) and initiated a 5-s timeout (TO) signaled by the illumination of the red, yellow, and green LEDs above the active lever and the houselight. Responding on the inactive lever and responding during TOs (active and inactive levers) were recorded but had no scheduled consequence. This schedule (FR1: TO $5 \mathrm{~s}$ ) was in place for 10 sessions, and all rats met acquisition criteria ( $>20$ infusions and $>80 \%$ of the responses emitted were on the active lever for 2 consecutive days). Subsequently, the response requirement increased to an FR5 for at least an additional 10 sessions, and upon meeting stability criteria $( \pm 20 \%$ of the mean number of infusions for 3 consecutive days, with no increasing or decreasing trend) all rats were transitioned to a progressive ratio (PR) schedule of reinforcement.

Reinforcing effectiveness of individual bath salts constituents. For the remainder of the experiment, responding was maintained under a PR schedule of reinforcement under which response requirements for each successive infusion incremented according to the following equation: ratio= $\left[5 \mathrm{e}^{(\mathrm{inf} \# \times 0.2)}\right]-5$. The maximum session duration was $12 \mathrm{~h}$, but sessions were terminated if a ratio was not completed within $45 \mathrm{~min}$ (ie, 45-min limited hold). Two groups of rats were trained to respond for $0.032 \mathrm{mg} / \mathrm{kg} / \mathrm{inf} \mathrm{MDPV}$, with one group $(n=12)$ used to evaluate MDPV (0.0032-0.32 mg/ 
$\mathrm{kg} /$ inf), caffeine $(0.1-1.78 \mathrm{mg} / \mathrm{kg} / \mathrm{inf})$, and their binary mixtures, and the other group $(n=19)$ used to evaluate MDPV (0.0032-0.32 mg/kg/inf), methylone $(0.1-1.78 \mathrm{mg} / \mathrm{kg} /$ inf), and their binary mixtures. A third group of rats $(n=15)$ was trained to respond for $0.32 \mathrm{mg} / \mathrm{kg} / \mathrm{inf}$ methylone and was used to evaluate methylone $(0.032-1.78 \mathrm{mg} / \mathrm{kg} / \mathrm{inf})$, caffeine $(0.1-1.78 \mathrm{mg} / \mathrm{kg} / \mathrm{inf})$, and their binary mixtures. The first dose evaluated was $0.032 \mathrm{mg} / \mathrm{kg} / \mathrm{inf} \mathrm{MDPV}$ (MDPV-trained rats) or $0.32 \mathrm{mg} / \mathrm{kg} / \mathrm{inf}$ methylone (methylone-trained rats), with all remaining doses evaluated in a random order. Each dose (or dose pair) was available for at least two consecutive sessions and until responding met stability criteria ( \pm 2 infusions from the previous session). All doses (dose pairs) for a particular drug (drug mixture) were evaluated prior to evaluating the next drug (drug mixture). Dose-response curves of individual constituents were established twice (once before and once after evaluation of drug mixtures) to determine whether responding changed over time. Because the study design required that 12 rats complete all portions of the study, and because toxicity was observed when rats were responding for methylone, seven rats were added to the MDPV+methylone group, and three rats were added to the methylone+caffeine group.

\section{Drug Mixtures}

Composition of binary bath salts mixtures. bath salts mixtures were constructed using the concept of doseequivalence (see Tallarida and Raffa, 2010). Accordingly, dose-response curves for individual subjects were first normalized to the dose condition that maintained the greatest number of infusions (ie, $E_{\max }$ ) and saline, with the number of infusions maintained by saline serving as the $0 \%$ effect level, and the difference in the number of infusions maintained by the $\mathrm{E}_{\max }$ and saline serving as the $100 \%$ effect level. Normalized dose-response curves were then fit using a linear regression of the data spanning the $20 \%-80 \%$ effect levels (no more than one data point $>80 \%$ and one data point $<20 \%$ ) to obtain $\mathrm{ED}_{50}$ s, slopes, and $\mathrm{y}$-intercepts for each pair of constituent drugs (ie, MDPV and caffeine, methylone and caffeine, or MDPV and methylone). Mixtures were designed at four ratios $(10: 1,3: 1,1: 1$, and $1: 3)$ relative to the mean $\mathrm{ED}_{50}$ for the group (ie, dose of each drug that maintained $50 \%$ of the maximal effect level). Since caffeine failed to maintain $50 \%$ of the maximum effect of either MDPV of methylone, the dose that maintained $50 \%$ of the maximal effect of caffeine $(0.56 \mathrm{mg} / \mathrm{kg} / \mathrm{inf})$ was used to determine the composition of MDPV+caffeine and methylone+caffeine mixtures. In order to fully evaluate the reinforcing effects of the bath salts mixtures, each fixed dose ratio mixture (eg, $10: 1,3: 1,1: 1$, or $1: 3$ ) included a series of fixed dose pairs that spanned the $0-100 \%$ predicted effect levels (see Supplementary Table 1 for more details).

Dose-addition analyses. Predicted additive effect levels for each dose pair were calculated by first converting the unit dose of each constituent into dose equivalents of the training drug (eg, caffeine into MDPV equivalents for the MDPV+caffeine mixtures) using the following function (equation 1) :

$\operatorname{DoseB}_{\mathrm{eq}} A=\left[\left(\right.\right.$ Slope $^{\mathrm{B}} \times$ Dose $\left.^{\mathrm{B}}\right)+\left(\right.$ int $^{\mathrm{B}}-$ int $\left.\left.^{\mathrm{A}}\right)\right] /$ Slope $^{\mathrm{A}}$ where Slope ${ }^{\mathrm{A}}$ and Slope ${ }^{\mathrm{B}}$ are the slope parameters and int ${ }^{\mathrm{A}}$ and in ${ }^{\mathrm{B}}$ are the $y$ intercepts derived from the linear portion of the dose-response curves of drugs A (the training drug) and $B$, respectively, and $\operatorname{Dose}^{B}$ is the unit dose of drug $B$ that is present in each dose pair. Summing the unit dose of drug A and the $\mathrm{B}_{\mathrm{eq}} \mathrm{A}$ allows each dose pair to be expressed in terms of the total dose equivalents of drug $A$. The total equivalent dose for each dose pair was then used to calculate the predicted effect for an additive interaction using the following function (equation 2):

Predicted additive effect level

$$
=\left(\text { Slope }^{\mathrm{A}} \times \text { Total dose }_{\mathrm{eq}} \mathrm{A}\right)+\text { int }^{\mathrm{A}}
$$

where total dose $\mathrm{eq}_{\mathrm{e}} \mathrm{A}$ is the total dose equivalents of drug $\mathrm{A}$ (ie, Dose $^{A}+D_{\text {Dose }} B_{e q} A$ from equation 1). Predicted additive doseresponse curves were determined for individual subjects for each drug mixture, and are graphically represented as the mean $( \pm$ SEM) of the total drug equivalents $(\mathrm{mg} / \mathrm{kg} / \mathrm{inf})$ and the mean $( \pm$ SEM) predicted effect level (normalized to the $E_{\max }$ for Drug A) for each fixed-dose pair of each drug mixture.

Statistical analyses. Dose-response curves for individual constituents were analyzed by one-way repeated measures ANOVA with post hoc Dunnett's tests. All dose-response curves were analyzed by linear regression to obtain measures of potency $\left(\mathrm{ED}_{50}\right)$ and effectiveness $\left(E_{\max }\right)$ in individual subjects. For each constituent drug, the $E_{\max }( \pm$ SEM) values were compared between groups (ie, MDPV in MDPV+ caffeine and MDPV+methylone groups, caffeine in MDPV + caffeine and methylone+caffeine groups, and methylone in $\mathrm{MDPV}+$ methylone and methylone+caffeine groups) of rats using a Student's $t$-test, while significant differences in $\mathrm{ED}_{50} \mathrm{~S}$ between groups were indicated by nonoverlapping $95 \%$ confidence intervals. For each bath salts mixture, potency (observed $\mathrm{ED}_{50} /$ predicted $\mathrm{ED}_{50}$ ) and effectiveness (predicted $E_{\text {max }} /$ observed $\mathrm{E}_{\text {max }}$ ) ratios were calculated for individual subjects at each of the four dose ratios $(10: 1,3: 1,1: 1$, and $1: 3$ ). Dose ratios for which the $95 \%$ confidence interval did not include 1 were considered to be significantly different than additive, with ratios less than 1 indicative of a supra-additive interaction, greater than 1 indicative of a sub-additive interaction, and inclusive of 1 indicative of an additive interaction.

\section{RESULTS}

\section{Reinforcing Effects of Individual Constituents}

Figure 1 (top row) shows the number of infusions maintained by the bath salts constituents alone for rats that responded for $\operatorname{MDPV}(\mathrm{F}(5,55)=231.0, p<0.001$; post hoc $0.01-0.178 \mathrm{mg} / \mathrm{kg} /$ inf $p<0.05)$ and caffeine $(\mathrm{F}(4,44)=15.1$, $p<0.001$; post hoc $1-1.78 \mathrm{mg} / \mathrm{kg} /$ inf $p<0.05$; left panels), methylone $(\mathrm{F}(5,55)=60.0, p<0.001$; post hoc $0.1-1.78 \mathrm{mg} /$ $\mathrm{kg} /$ inf $p<0.05)$, and caffeine $(\mathrm{F}(4,44)=23.5, p<0.001$; post hoc $1-1.78 \mathrm{mg} / \mathrm{kg} /$ inf $p<0.05$; middle panels) and MDPV $(\mathrm{F}(5,70)=259.3, \quad p<0.001$; post hoc $0.01-0.178 \mathrm{mg} / \mathrm{kg} / \mathrm{inf}$ $p<0.05)$ and methylone $(\mathrm{F}(4,56)=96.6, p<0.001$; post hoc $0.32-1.78 \mathrm{mg} / \mathrm{kg} / \mathrm{inf} \quad p<0.05$; right panels). Normalized dose-response curves for the self-administration of bath 

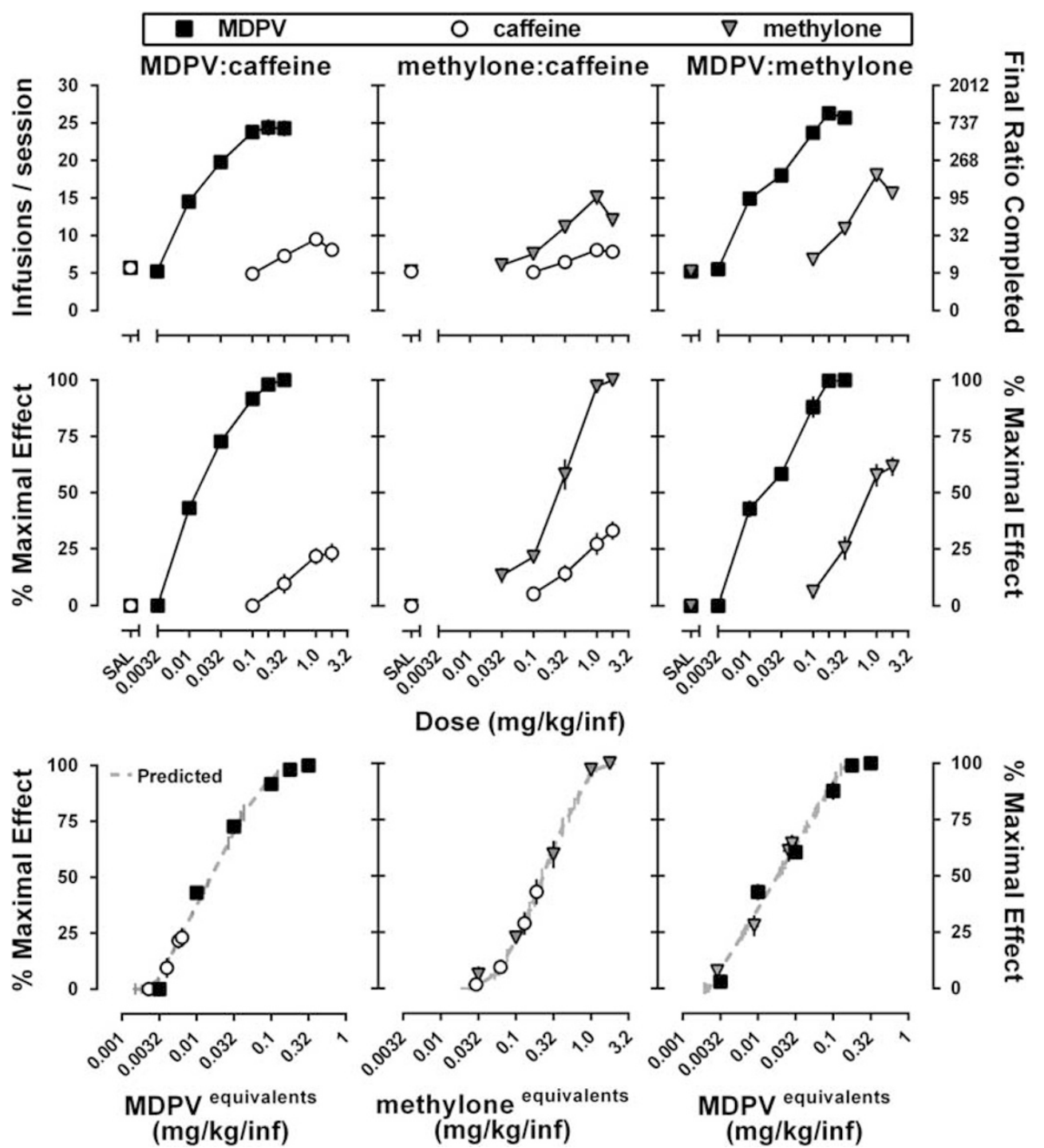

Figure I Dose-response curves for the self-administration of MDPV (squares), caffeine (circles), and methylone (triangles) under a progressive ratio schedule of reinforcement ( $n=12$ per group). Abscissa: first and second rows - 'SAL' represents data obtained when saline was available for infusion, whereas doses refer to the unit dose of each drug available for infusion expressed as mg/kg/inf on a log scale. Third row — doses refer to the MDPV (first and third columns) or methylone (middle column) equivalents available for infusion expressed as $\mathrm{mg} / \mathrm{kg} / \mathrm{inf}$ on a log scale. Ordinate: first row-total infusions $\pm \mathrm{SEM}$ obtained during the session. Second and third rows - percent of the maximal effect \pm SEM, normalized to the drug in each pair that maintained the most responding (100\%) and saline (0\%).

salts constituents are shown in the lower two panels of Figure 1. All three bath salts constituents maintained responding in a dose-dependent manner; however, differences in potency (rank order: $\mathrm{MDPV}>$ methylone $=$ caffeine) and effectiveness (rank order: MDPV $>$ methylone $>$ caffeine; maximum number of infusions) were observed. Because each drug was evaluated in two groups of rats, between-group comparisons of maximum number of infusions earned $\left(E_{\max }\right)$ and potency $\left(\mathrm{ED}_{50}\right)$ were also made. Although the $E_{\max }$ for MDPV $(26.4 \pm 1.0$ for MDPV+caffeine and $26.3 \pm 1.0$ for MDPV+methylone) and caffeine $(8.7 \pm 0.4$ for MDPV+caffeine and $8.3 \pm 0.5$ for methylone+caffeine) were comparable between groups (Table 1), methylone maintained significantly less responding $(t=2.77, p<0.05)$ in rats from the methylone+caffeine group $(14.8 \pm 0.8)$ than in rats from the MDPV+methylone group (18.1 \pm 0.9$)$. Differences in potency were not observed for any constituent. For the majority of the drugs, slope, $\mathrm{ED}_{50}$, and $E_{\max }$ values did not differ for dose-response curves generated before and after evaluation of mixtures; however, for rats from the MDPV+ caffeine group, caffeine's effects ( $E_{\max }$ and slope) were significantly decreased upon redetermination (data not shown).

Self-administration of methylone alone $(1.0$ and $1.78 \mathrm{mg} /$ $\mathrm{kg} / \mathrm{inf}$ ) was lethal in 2 of 19 MDPV-trained rats and 1 of 15 methylone-trained rats.

\section{Reinforcing Effects of Binary Mixtures of MDPV and Caffeine}

Predicted and observed dose-response curves for mixtures of MDPV+caffeine are shown in Figure 2 (top row). When 
Table I Reinforcing Effectiveness of bath salts Constituents MDPV, Methylone, and Caffeine Alone and in Binary Mixtures

\begin{tabular}{|c|c|c|c|c|c|c|}
\hline & \multicolumn{2}{|c|}{ MDPV : caffeine } & \multicolumn{2}{|c|}{ Methylone : caffeine } & \multicolumn{2}{|c|}{ MDPV : methylone } \\
\hline & $E_{\max }( \pm$ SEM $)$ & Final ratio $( \pm$ SEM $)$ & $E_{\max }( \pm$ SEM $)$ & Final ratio $( \pm$ SEM) & $E_{\max }( \pm$ SEM $)$ & Final ratio $( \pm$ SEM) \\
\hline Constituent 2 & $8.7(0.4)$ & $27(3)$ & $8.3(0.5)$ & $24(3)$ & | $8.1(0.9)$ & $27 \mid(70)$ \\
\hline I0: I Mixture & $25.5(0.8)$ & $947(156)$ & $16.2(0.7)$ & $138(20)$ & $24.1(1.5)$ & $1007(350)$ \\
\hline I : 3 Mixture & $19.3(0.6)$ & $256(30)$ & $12.8(0.4)$ & $62(6)$ & I $6.7(0.9)$ & $169(36)$ \\
\hline
\end{tabular}
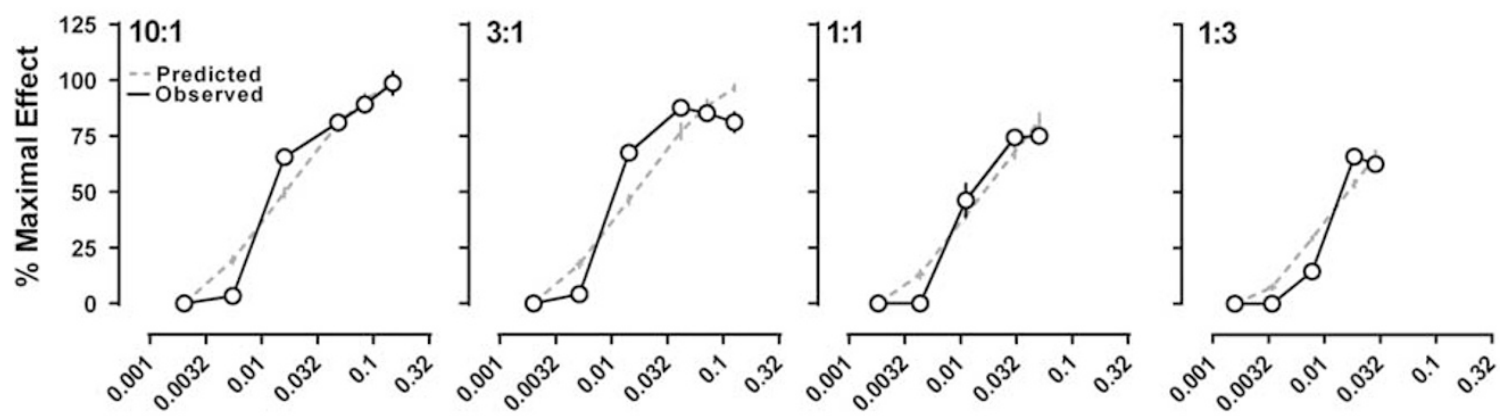

total MDPV equivalents ( $\mathrm{mg} / \mathrm{kg} / \mathrm{inf})$
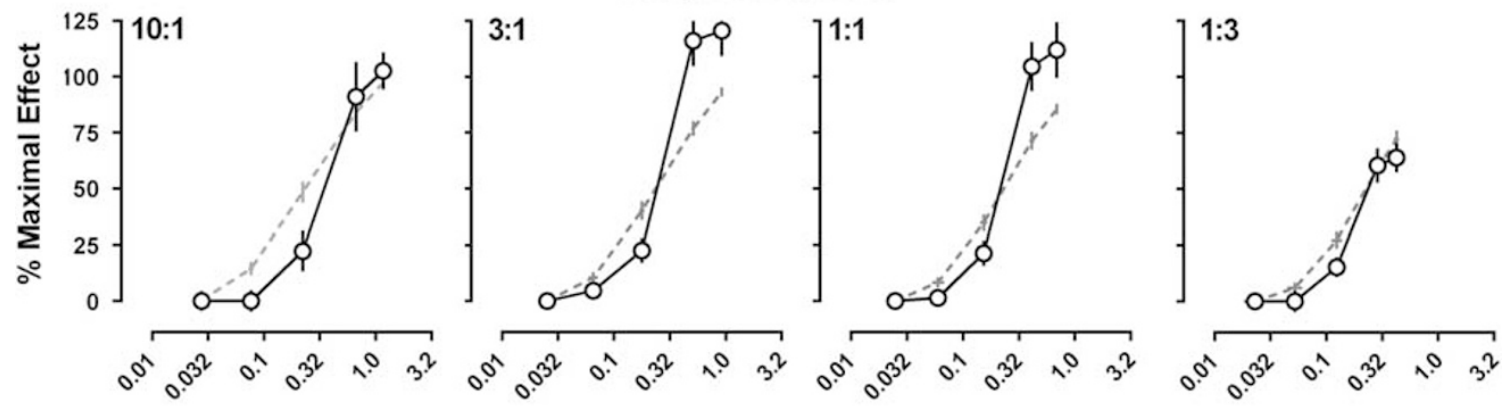

total methylone equivalents ( $\mathrm{mg} / \mathrm{kg} / \mathrm{inf})$

MDPV : methylone
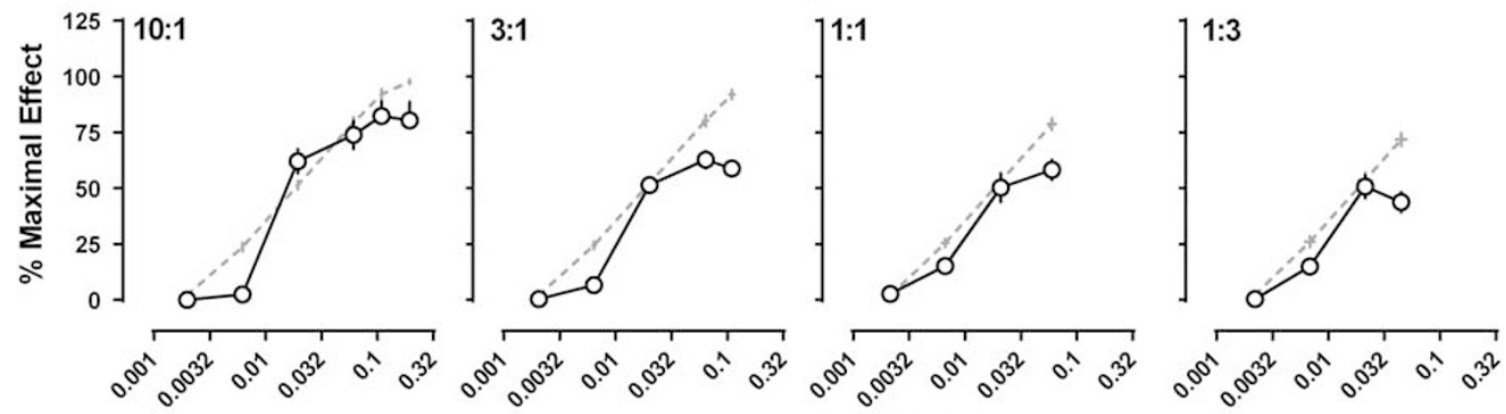

total MDPV equivalents $(\mathrm{mg} / \mathrm{kg} / \mathrm{inf})$

Figure 2 Dose-response curves for the self-administration of binary mixtures of MDPV+caffeine (top row), methylone+caffeine (middle row), or MDPV +methylone (bottom row; $n=12$ per group). Each mixture was tested at four fixed-dose ratios ( I $0:$ I (left column), $3:$ I (left-center column), I : I (rightcenter column), I:3 (right column)) relative to the $\mathrm{ED}_{50}$ of the drug in each pair that maintained the most responding. Experimentally determined doseresponse curves (white circles) represent the mean ( \pm SEM) for 12 rats. Predicted, additive dose-response curves (gray, dashed lines) represent the mean ( \pm SEM) for 12 rats. Abscissa: doses refer to total MDPV (top and bottom rows) or methylone (middle row) equivalents available for infusion expressed as mg/kg/ inf on a log scale. Ordinate: percent of the maximal effect, normalized to the drug in each pair that maintained the most responding (I00\%), and saline (0\%) as shown in Figure I. 
combined at dose ratios of $10: 1,3: 1,1: 1$, and $1: 3$, mixtures of MDPV+caffeine maintained responding in dose-dependent manner. With the exception of the $3: 1$ MDPV : caffeine ratio, the dose-response curves obtained for mixtures of MDPV+caffeine did not depart from the predicted additive dose-response curves. Although the $3: 1$ mixture of MDPV+caffeine was significantly more potent than predicted (Figure 3; top row, left panel), none of the mixtures of MDPV and caffeine differed from predictions for a strictly additive interaction with regard to their reinforcing effectiveness (Figure 3; top row, right panel). The $E_{\max } \pm$ SEM and the final ratio completed \pm SEM for each mixture of MDPV+caffeine are reported in Table 1 .

\section{Reinforcing Effects of Binary Mixtures of Methylone and Caffeine}

Predicted and observed dose-response curves for mixtures of methylone and caffeine are also shown in Figure 2 (middle row). As with mixtures of MDPV+caffeine, dose-dependent increases in responding were observed for all fixed-dose ratios of methylone+caffeine. Although each of the mixtures exhibited additive interactions with respect to potency (Figure 3; middle row, left panel), when evaluated at $3: 1$ and $1: 1$ ratios of methylone: caffeine, the two largest dose pairs of each mixture consistently maintained more responding than predicted for an additive interaction. Indeed, the effectiveness ratios for the $3: 1$ and $1: 1$ mixtures of methylone+caffeine were significantly smaller than 1 , indicating a supra-additive interaction between methylone and caffeine with regard to their reinforcing effectiveness (Figure 3; middle row, right panel). The $E_{\max } \pm$ SEM and the final ratio completed \pm SEM for each mixture of methylone+ caffeine are reported in Table 1.

Self-administration of the largest dose pair of the $3: 1$ mixture of methylone+caffeine $(0.80 \mathrm{mg} / \mathrm{kg} / \mathrm{inf}$ methylone $+0.79 \mathrm{mg} / \mathrm{kg} /$ inf caffeine) was lethal in 2 out of 14 rats.

\section{Reinforcing Effects of Binary Mixtures of MDPV and Methylone}

As observed with other binary bath salts mixtures, mixtures of MDPV and methylone maintained dose-dependent increases in self-administration, regardless of the ratio at which the cathinones were mixed (Figure 2; bottom row). Although the reinforcing effects of small dose pairs of $\mathrm{MDPV}+$ methylone appeared to be additive in nature, larger dose pairs at the $3: 1,1: 1$, and $1: 3$ ratios of MDPV + methylone tended to maintain less responding than predicted for an additive interaction. Indeed, although substantial variability was observed among the potency ratios for the MDPV+methylone group, the 3:1 mixture of MDPV+ methylone exhibited a sub-additive interaction with regard to potency; additive interactions were observed for all other mixtures (Figure 3; bottom row, left panel). With regard to reinforcing effectiveness, an additive interaction was observed for the 10:1 ratio, whereas the effectiveness ratios for the $3: 1,1: 1$, and $1: 3$ mixtures of MDPV+methylone were all significantly greater than 1 , indicative of a subadditive interaction (Figure 3; bottom row, right panel). The $E_{\max } \pm$ SEM and the final ratio completed \pm SEM for each mixture of MDPV+methylone are reported in Table 1.

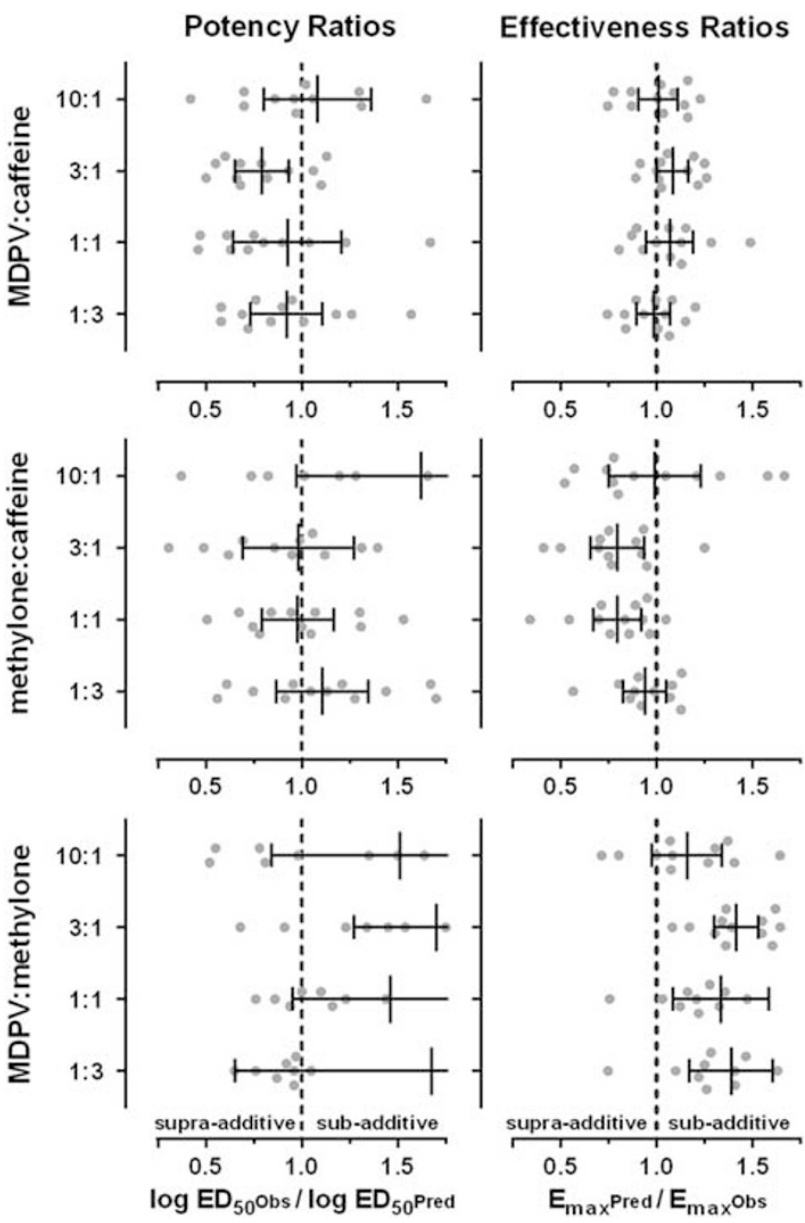

Figure 3 Potency ratios (observed $\mathrm{ED}_{50}$ /predicted additive ED 50 , left column) and effectiveness ratios (predicted additive $E_{\max }$ /observed $E_{\max }$, right column) for binary mixtures of MDPV+caffeine (top row), methylone + caffeine (middle row), and MDPV+methylone (bottom row; $n=12$ per group). Ratios for individual subjects are depicted by the gray dots. The group means and $95 \%$ confidence intervals are depicted by the vertical black line and error bars, respectively.

The self-administration of MDPV+methylone mixtures was associated with lethality in 5 of 17 rats. For the $1: 1$ mixture, 1 of 14 rats died at the fourth dose pair $(0.03 \mathrm{mg} / \mathrm{kg} /$ inf MDPV $+1.23 \mathrm{mg} / \mathrm{kg} / \mathrm{inf}$ methylone), and 2 of 4 rats died at the fifth dose pair $(0.05 \mathrm{mg} / \mathrm{kg} / \mathrm{inf} \mathrm{MDPV}+2.19 \mathrm{mg} / \mathrm{kg} / \mathrm{inf}$ methylone). At the $1: 3$ mixture, 1 of 14 rats died at the fourth dose pair $(0.015 \mathrm{mg} / \mathrm{kg} /$ inf $\mathrm{MDPV}+1.85 \mathrm{mg} / \mathrm{kg} / \mathrm{inf}$ methylone), and 1 of 2 rats died at the fifth dose pair (0.03 mg/kg/inf MDPV, $3.3 \mathrm{mg} / \mathrm{kg} /$ inf methylone). Because of the high incidence of lethality (50\%), the largest dose pairs of the $1: 1$ and $1: 3$ mixtures of MDPV+methylone were not evaluated in all subjects.

\section{DISCUSSION}

bath salts preparations typically contain mixtures of drugs including multiple synthetic cathinones or synthetic cathinones and caffeine, and use of these preparations is associated with high rates of abuse, toxicity, and death. Despite this, little is known about how the composition of these bath salts preparations has an impact on their abuse- 
related and toxic effects. As such, the current study evaluated the reinforcing effects of three common bath salts constituents (ie, MDPV, methylone, and caffeine) and used dose-addition analyses to characterize the nature of the interaction(s) between binary bath salts mixtures comprising two synthetic cathinones (ie, MDPV+methylone) or a synthetic cathinone and caffeine (ie, MDPV+caffeine and methylone+caffeine). The present study provides evidence that the composition of bath salts preparations can have a significant impact on both their reinforcing potency and effectiveness, with mixtures of MDPV+caffeine $(3: 1)$ being more potent than predicted for an additive interaction, mixtures of methylone+caffeine $(3: 1$ and $1: 1)$ being more effective than predicted for an additive interaction, and mixtures of MDPV+methylone $(3: 1,1: 1$, and $1: 3)$ being less effective and less potent $(3: 1)$ than predicted for an additive interaction.

Consistent with literature describing the reinforcing effects of MDPV (Aarde et al, 2013; Watterson et al, 2014; Schindler et al, 2016; Gannon et al, 2017a; Gannon et al, 2017b), methylone (Watterson et al, 2013; Creehan et al, 2015; Vandewater et al, 2015; Nguyen et al, 2016), and caffeine alone (Collins et al, 1984; Briscoe et al, 1998), MDPV was the most effective, whereas caffeine was the least effective of the bath salts constituents. Importantly, although numerous studies have used $\mathrm{PR}$ schedules to compare relative reinforcing effectiveness of MDPV and methylone to other stimulants (eg, cocaine, methamphetamine, and MDMA), this is the first to directly compare these two common bath salts constituents. Using the final ratio completed as an index of relative reinforcing effectiveness, MDPV (1123.2 \pm 114.1$)$ was an $\sim 6$-fold more effective reinforcer than methylone $(190.4 \pm 39.4)$ and an $\sim 45$-fold more effective reinforcer than caffeine $(24.7 \pm 1.9)$. Although previous reports suggest that the reinforcing effects of IV caffeine are dubious and shortlived (Atkinson and Enslen, 1976; Briscoe et al, 1998), in the present study, caffeine maintained low but consistent levels of responding when substituted from a MDPV or methylone baseline, regardless of the order that the doses were evaluated. However, upon re-evaluation at the end of the study, caffeine appeared to be less effective in the MDPVtrained rats.

Even though caffeine was found to be a weak reinforcer, supra-additive interactions were observed when caffeine was self-administered in combination with more effective reinforcers. For instance, when mixed at a 3:1 ratio with MDPV, a highly effective reinforcer, caffeine produced a supra-additive interaction with regard to reinforcing potency, suggesting that bath salts preparations containing MDPV and caffeine can produce a full MDPV-like effect with significantly less MDPV. Conversely, when mixed at a $3: 1$ or $1: 1$ ratio with methylone, a moderately effective reinforcer, caffeine produced a supra-additive interaction with regard to reinforcing effectiveness, suggesting that the reinforcing effects of bath salts preparations containing methylone and caffeine would be significantly greater than bath salts preparations containing only methylone. Although these studies were not designed to identify the mechanism(s) that underlie interactions between caffeine and the synthetic cathinones, caffeine (through its antagonism of adenosine $\mathrm{A}_{2 \mathrm{~A}}$ receptors) can increase dopamine $\mathrm{D}_{2}$ receptor signaling, which has been hypothesized to have a role in caffeine's ability to potentiate the psychostimulant effects of other indirect dopamine receptor agonists (eg, cocaine; Ferré, 2016). Although it is unclear why the effects of caffeine differed in bath salts mixtures containing caffeine+MDPV (increased potency) and caffeine+methylone (increased effectiveness), it is a possibility that a ceiling effect limited our ability to detect enhancements in the effectiveness for mixtures of MDPV+caffeine, and that supra-additive interactions would have been observed if different methods for quantifying reinforcing effectiveness had been employed (eg, demand curve analyses). Alternatively, it is also possible that differences in the types of supra-additive interactions are related to differences in the mechanism of action for MDPV (highly selective inhibitor of DAT) and methylone (nonselective substrate at DAT, NET, and SERT; Baumann et al, 2013; Simmler et al, 2013). Clearly, more in-depth investigations into the mechanisms that underlie these interactions are warranted.

Whereas supra-additive interactions were observed for bath salts mixtures containing caffeine, sub-additive interactions were observed when MDPV and methylone were combined. Although the 3:1 mixture of MDPV+methylone was found to be less potent than predicted for an additive interaction, $3: 1$, $1: 1$, and $1: 3$ mixtures of MDPV+methylone were all found to exhibit sub-additive interactions with regard to reinforcing effectiveness. This finding contrasts a report that identified a supra-additive interaction between mephedrone (a monoamine transporter substrate) and MDPV with regard to excitatory hDAT currents, and proposed that this could result in mixtures of substrates and inhibitors producing effects greater than either drug alone (Cameron et al, 2013). However, it is important to note that methylone, and MDPV to a lesser extent, also has actions at NET and SERT that likely have an impact on the reinforcing effects of these drugs/drug mixtures that are not captured in an isolated in vitro system. Interestingly, as the proportion of methylone in the MDPV + methylone mixtures increased, the maximal effect observed became more similar to the maximal effect produced by methylone alone (see Figure 1, bottom right panel). Thus, one interpretation of these findings is that the actions of methylone at SERT may be decreasing the reinforcing effectiveness of the MDPV+methylone mixture, a notion that is supported by the demonstration that increases in serotoninergic activity are associated with decreases in stimulant self-administration by nonhuman primates (Wee et al, 2005; Wee and Woolverton, 2006).

However, because these sub-additive interactions were observed at larger dose pairs, it is also possible that decreases in reinforcing effectiveness resulted from the onset of other/ adverse effects that limited the ability of the rats to respond at high rates. Indeed, the self-administration of these large dose pairs was lethal in five rats. Although the mechanism of this toxicity is unclear, it is likely related to the fact that methylone is a mechanism-based inhibitor of CYP2D6, the enzyme primarily responsible for the metabolism of methylone (Pederson et al, 2013; Elmore et al, 2017). However, because lethality has not previously been reported for rats self-administering methylone (eg, Watterson et al, 2013; Creehan et al, 2015; Vandewater et al, 2015; Nguyen et al, 2016), and because the majority of the deaths (7 of 10) were associated with the self-administration of bath salts mixtures containing methylone (five with methylone+ 
MDPV and two with methylone+caffeine), pharmacodynamic interactions between the toxic effects of bath salts constituents cannot be ruled out. Importantly, methylone has also been associated with toxicity and death in human bath salts users, particularly when used in preparations that also contain MDPV (eg, Spiller et al, 2011; Pearson et al, 2012). Since MDPV maintains very high levels of responding without overt signs of toxicity, and toxicity was only observed in the presence of methylone, it is likely that the toxicity observed with mixtures of MDPV+methylone was the result of MDPV maintaining high rates of responding that also resulted in large cumulative doses of methylone.

Abuse and toxicity associated with the use of bath salts has become a major public concern in the past decade. While it has been demonstrated that some common constituents of these preparations (eg, MDPV) are more effective than other drugs of abuse when self-administered alone (eg, Gannon et al, 2017a), bath salts preparations are often mixtures of multiple drugs. The present study is the first to directly assess the reinforcing effects of mixtures of common bath salts constituents. There were four main findings: (1) MDPV is a more potent and effective reinforcer than methylone, which is equipotent but more effective than caffeine; (2) bath salts mixtures containing caffeine can function as more potent (MDPV+caffeine) and more effective (methylone+caffeine) reinforcers than expected based on the effects of the constituents alone; (3) bath salts mixtures containing $\mathrm{MDPV}+$ methylone are less effective reinforcers than expected; and (4) bath salts mixtures containing methylone appear to be more toxic than methylone alone. Although most bath salts mixtures exhibited strictly additive interactions, these findings indicate that supra-additive interactions can occur between drugs with different mechanisms of action (ie, MDPV+caffeine and methylone+caffeine) and sub-additive interactions can occur between drugs that act primarily at monoamine transporters but differ in transporter selectivity (ie, MDPV+methylone). Taken together, these studies demonstrate that the composition of bath salts preparations can have a significant impact on their abuserelated and toxic effects, and suggest that such interactions could contribute to the patterns of use and/or the adverse effects reported by human bath salts users.

\section{FUNDING AND DISCLOSURE}

This research was supported by grants from the National Institutes of Health and the National Institute on Drug Abuse (R01DA039146 (GTC), T32DA031115 (BMG)). The work of the Molecular Targets and Medications Discovery Branch was supported by the Intramural Research Programs of the National Institute on Drug Abuse and the National Institute of Alcohol Abuse and Alcoholism. The authors declare no conflict of interest.

\section{REFERENCES}

Aarde SM, Huang PK, Creehan KM, Dickerson TJ, Taffe MA (2013). The novel recreational drug 3,4-methylenedioxypyrovalerone (MDPV) is a potent psychomotor stimulant: selfadministration and locomotor activity in rats. Neuropharmacology 71: 130-140.
Atkinson J, Enslen M (1976). Self-administration of caffeine by the rat. Arzneimittelforschung 26: 2059-2061.

Baumann MH, Partilla JS, Lehner KR, Thorndike EB, Hoffman AF, Holy $M$ et al (2013). Powerful cocaine-like actions of 3, 4-methylenedioxypyrovalerone (MDPV), a principal constituent of psychoactive bath salts products. Neuropsychopharmacology 38: $552-562$.

Brandt SD, Sumnall HR, Measham F, Cole J (2010). Analyses of second-generation 'legal highs' in the UK: Initial findings. Drug Test Anal 2: 377-382.

Briscoe RJ, Vanecek SA, Vallett M, Baird TJ, Holloway FA, Gauvin DV (1998). Reinforcing effects of caffeine, ephedrine, and their binary combination in rats. Pharmacol Biochem Behav 60: 685-693.

Cameron KN, Kolanos R, Solis E Jr, Glennon RA, De Felice LJ (2013). Bath salts components mephedrone and methylenedioxypyrovalerone (MDPV) act synergistically at the human dopamine transporter. Br J Pharmacol 168: 1750-1757.

Caudevilla-Gálligo F, Ventura M, Iciar BI, Ruiz I, Fornís I (2013). Presence and composition of cathinone derivatives in drug samples taken from a Drug Test Service in Spain (2010-2012). Hum Psychopharmacol 28: 341-344.

Collins RJ, Weeks JR, Cooper MM, Good PI, Russell RR (1984). Prediction of abuse liability of drugs using IV self-administration by rats. Psychopharmacology 82: 6-13.

Collins GT, Abbott M, Galindo K, Rush EL, Rice KC, France CP (2016). Discriminative stimulus effects of binary drug mixtures: studies with cocaine, MDPV, and caffeine. J Pharmacol Exp Ther 359: $1-10$.

Comer SD, Carroll ME (1996). Oral caffeine pretreatment produced modest increases in smoked cocaine self-administration in rhesus monkeys. Psychopharmacology 126: 281-285.

Creehan KM, Vandewater SA, Taffe MA (2015). Intravenous selfadministration of mephedrone, methylone, and MDMA in female rats. Neuropharmacology 92: 90-97.

Davies S, Wood DM, Smith G, Button J, Ramsey J, Archer R et al (2010). Purchasing "legal highs" on the Internet-is there consistency in what you get? Q J Med 103: 489-493.

Elmore JS, Dillon-Carter O, Partilla JS, Ellefsen KN, Concheiro M, Suzuki $M$ et al (2017). Pharmacokinetic profiles and pharmacodynamics effects for methylone and its metabolites in rats. Neuropsychopharmacology 42: 649-660.

Eshleman AJ, Wolfrum KM, Hatfield MG, Johnson RA, Murphy KV, Janowsky A (2013). Substituted methcathinones differ in transporter and receptor interactions. Biochem Pharmacol 85: $1803-1815$.

Ferré S (2016). Mechanisms of the psychostimulant effects of caffeine: implications for substance use disorders. Psychopharmacology 233: 1963-1973.

Forrester MB (2012). Synthetic cathinone exposures reported to Texas poison centers. Am J Drug Alcohol Abuse 38: 609-615.

Gannon BM, Galindo KI, Rice KC, Collins GT (2017a). Individual differences in the relative reinforcing effects of 3,4-methylenedioxypyrovalerone (MDPV) under fixed and progressive ratio schedules of reinforcement in rats. J Pharmacol Exp Ther 361: 181-189.

Gannon BM, Rice KC, Collins GT (2017b). Reinforcing effects of 3,4-methylenedioxypyrovalerone (MDPV), $\alpha$-pyrrolidinopentiophenone ( $\alpha$-PVP) and their enantiomers in rats. Behav Pharmacol (e-pub ahead of print 31 May 2017; doi:10.1097/ FBP.0000000000000315).

Garrett BE, Griffiths RR (2001). Intravenous nicotine and caffeine: subjective and physiological effects in cocaine abusers. J Pharmacol Exp Ther 296: 486-494.

Goh CY, van Bronswijk W, Priddis C (2008). Rapid nondestructive on-site screening of methylamphetamine seizures by attenuated total reflection fourier transform infrared spectroscopy. Appl Spectrosc 62: 640-648. 
Horger BA, Wellman PJ, Morien A, Davies BT, Schenk S (1991). Caffeine exposure sensitizes rats to the reinforcing effects of cocaine. Neuroreport 2: 53-56.

Johnson PS, Johnson MW (2014). Investigation of "bath salts" use patterns within an online sample of users in the United States. J Psychoactive Drugs 46: 369-378.

Justinova Z, Ferré S, Barnes C, Wertheim CE, Pappas LA, Goldberg SR et al (2009). Effects of chronic caffeine exposure on adenosinergic modulation of the discriminative-stimulus effects of nicotine, methamphetamine, and cocaine in rats. Psychopharmacology 203: 355-367.

Justinova Z, Ferré S, Segal PN, Antoniou K, Solinas M, Pappas LA et al (2003). Involvement of adenosine $A 1$ and $A 2 A$ receptors in the adenosinergic modulation of the discriminative-stimulus effects of cocaine and methamphetamine in rats. J Pharmacol Exp Ther 307: 977-986.

Lapachinske SF, Okai GG, dos Santos A, de Bairros AV, Yonamine M (2015). Analysis of cocaine and its adulterants in drugs for international trafficking seized by the Brazilian Federal Police. Forensic Sci Int 247: 28-53.

National Research Council (2011). Guide for the Care and Use of Laboratory Animals8th ednNational Academy Press: Washington, DC, USA.

Nguyen JD, Grant Y, Creehan KM, Vandewater SA, Taffe MA (2016). Escalation of intravenous self-administration of methylone and mephedrone under extended access conditions. Addict Biol (e-pub ahead of print 5 April 2016; doi:10.1111/adb.12398).

Pearson JM, Hargraves TL, Hair LR, Massucci CJ, Frazee CC III, Garg U et al (2012). Three fatal intoxications due to methylone. J Anal Toxicol 36: 444-451.

Pederson AJ, Petersen TH, Linnet K (2013). In vitro metabolism and pharmacokinetic studies on methylone. Drug Metab Dispos 41: 1247-1255.

Ross EA, Watson M, Goldberger B (2011). "Bath salts" intoxication. $N$ Engl J Med 365: 967-968.

Schenk S, Valadez A, Horger BA, Snow S, Wellman PJ (1994). Interactions between caffeine and cocaine in tests of selfadministration. Behav Pharmacol 5: 153-158.

Schindler CW, Thorndike EB, Goldberg SR, Lehner KR, Cozzi NV, Brandt SD et al (2016). Reinforcing and neurochemical effects of the "bath salts" constituents 3,4-methylenedioxypyrovalerone (MDPV) and 3,4-methylenedioxy-N-methylcathinone (methylone) in male rats. Psychopharmacology 233: 1981-1990.

Seely KA, Patton AL, Moran CL, Womack ML, Prather PL, Fantegrossi WE et al (2013). Forensic investigation of K2, Spice, and "bath salt" commercial preparations: a three-year study of new designer drug products containing synthetic cannabinoid, stimulant, and hallucinogenic compounds. Forensic Sci Int 233: 416-422.

Shanks KG, Dahn T, Behonick G, Terrell A (2012). Analysis of first and second generation legal highs for synthetic cannabinoids and synthetic stimulants by ultra-performance liquid chromatography and time of flight mass spectrometry. J Anal Toxicol 36: 360-371.

Simmler LD, Buser TA, Donzelli M, Schramm Y, Dieu L-H, Huwyler J et al (2013). Pharmacological characterization of designer cathinones in vitro. Br J Pharmacol 168: 458-470.

Spiller HA, Ryan ML, Weston RG, Jansen J (2011). Clinical experience with and analytical confirmation of bath salts and "legal highs" (synthetic cathinones) in the United States. Clin Toxicol 49: 499-505.

Substance Abuse and Mental Health Services Administration. Drug Abuse Warning Network, 2011: National Estimates of Drug-Related Emergency Department Visits. HHS Publication No. (SMA) 13-4760, DAWN Series D-39. Substance Abuse and Mental Health Services Administration: Rockville, MD, 2013.

Tallarida RJ, Raffa RB (2010). The application of drug dose equivalence in the quantitative analysis of receptor occupation and drug combinations. Pharmacol Ther 127: 165-174.

Vandewater SA, Creehan KM, Taffe MA (2015). Intravenous selfadministration of entactogen-class stimulants in male rats. Neuropharmacology 99: 538-545.

Vidal Giné C, Ventura Vilamala M, Fornís Espinosa I, Gil Lladanosa C, Calzada Álvarez N, Fitó Fruitós A et al (2016). Crystals and tablets in the Spanish ecstasy market 2000-2014: are they the same or different in terms of purity and adulteration? Forensic Sci Int 263: 164-168.

Watterson LR, Hood L, Sewalia K, Tomek SE, Yahn S, Johnson CT et al (2013). The reinforcing and rewarding effects of methylone, a synthetic cathinone commonly found in "bath salts". J Addict Res Ther Supp 9: 002. doi:10.4172/2155-6105.S9-002.

Watterson LR, Kufahl PR, Nemirovsky NE, Sewalia K, Grabenauer M, Thomas BF et al (2014). Potent rewarding and reinforcing effects of the synthetic cathinone 3,4-methylenedioxypyrovalerone (MDPV). Addict Biol 19: 165-174.

Wee S, Anderson KG, Baumann MH, Rothman RB, Blough BE, Woolverton WL (2005). Relationship between the serotonergic activity and reinforcing effects of a series of amphetamine analogs. J Pharmacol Exp Ther 313: 848-854.

Wee S, Woolverton WL (2006). Self-administration of mixtures of fenfluramine and amphetamine by rhesus monkeys. Pharmacol Biochem Behav 84: 337-343.

Zuba D, Byrska B (2013). Prevalence and co-existance of active components of "legal highs". Drug Test Anal 5: 420-429.

Supplementary Information accompanies the paper on the Neuropsychopharmacology website (http://www.nature.com/npp) 